\title{
miR-590-3p Alleviates diabetic peripheral neuropathic pain by targeting RAP1A and suppressing infiltration by the $T$ cells
}

\author{
Yihua Wü\#, Ye Gu# and Bimin Shi' ${ }^{1 \otimes}$ \\ 'Department of Endocrinology, The First Affiliated Hospital of Soochow University, Suzhou City, Jiangsu Province, 215000, China; 2 Department \\ of Endocrinology, Shanghai International Medical Center, Shanghai, 200318, China
}

Background: MicroRNAs play a crucial role in diabetic peripheral neuropathic pain (DPNP). miR-590-3p is a novel miRNA and involved in multiple diseases. However, the pathological mechanism of miR-590-3p in DPNP needs to be elucidated. Materials and methods: The $\mathrm{db} / \mathrm{db}$ mice and $\mathrm{db} / \mathrm{m}$ mice were selected to mimic diabetes and control, respectively, for in vivo studies. The miR-590-3p agomir was injected into $\mathrm{db} / \mathrm{db}$ mice and pain-related behavioral tests were performed. The interaction of miR-590-3p with target gene was confirmed by dual-luciferase reporter assay. The expression of target gene was determined by qRT-PCR and western blot assay. The levels of inflammatory cytokines were measured by enzyme-linked immunosorbent assay (ELISA). Results: miR-590-3p was down-regulated in diabetic peripheral neuropathy mice. More importantly, miR-590-3p agomir alleviated pain-related behavior, reduced TNF-a, IL-1 $\beta$ and IL- 6 concentrations, and inhibited neural infiltration by immune cells in $\mathrm{db} / \mathrm{db}$ mice. Interestingly, RAP1A was predicted to be the target of miR-590-3p by Targetscan, and was actually regulated by miR-590-3p. Finally, the rescue experiments proved that overexpression of RAP1A partially abrogated the suppressive impact of $m i R-590-3 p$ on T cells proliferation and migration. Conclusion: miR-590-3p ameliorates DPNP via targeting RAP1A and inhibiting $\mathrm{T}$ cells infiltration, indicating that exogenous $m i R-590-3 p$ may be a potential candidate for clinical treatment of DPNP.

Key words: miR-590-3p, diabetic peripheral neuropathic pain, proinflammatory cytokines, T cell

Received: 13 August, 2020; revised: 24 September, 2020; accepted: 30 October, 2020; available on-line: 17 December, 2020

घe-mail: bmshi666@163.com

\#These authors contributed equally to the work.

Abbreviations: DPNP, diabetic peripheral neuropathic pain; DRG, dorsal root ganglion; PN, diabetic peripheral neuralgia; ELISA, enzyme-linked immunosorbent assay; IHC, immunohistochemistry; IL-1 $\beta$, interleukin-1 beta; SDS-PAGE, sodium dodecyl sulfate polyacrylamide gel electrophoresis; TBST, tris buffered saline with Tween; TNF-a, tumor necrosis factor alpha

\section{INTRODUCTION}

Diabetes has a high prevalence rate and has become a common chronic disease all over the world. It also induces a complication - diabetic neuropathy, which causes diabetic peripheral neuropathic pain (DPNP) (Davies et al., 2006). Diabetic patients with DPNP experience limb numbness, spontaneous pain, hyperalgesia, or allodynia (Calcutt 2004). Given that diabetic peripheral neuralgia (DPN) is a typical chronic neurogenic pain, it is difficult to treat, affects the physiological functions and quality of life of the patients, and brings a heavy burden to individuals and society (Boulton et al., 2004). The pathogenesis of DPNP is complex, including inflammation, repair process, and gene expression (Vanotti et al., 2007). Although the diverse treatment strategies have advanced, DPNP is still difficult to cure. Therefore, the pathogenesis of DPNP should be further clarified and new treatment strategies should be developed.

MicroRNA (miR), is a non-coding RNA, which generally has a length of 18-22-nucleotides. MiRs can bind to the 3'-UTR of the target gene to modulate the gene expression (Wu et al., 2013). Currently, the expression of miRs has been proved to be closely related to DPN. miR-146a influences the severity of DPN through the regulation of inflammation (Feng et al., 2018). Moreover, knockdown of miR-25 can significantly aggravate diabetic peripheral neuralgia via the production of the reactive oxygen species (Zhang et al., 2018). Overexpression of miR$146 a$ can suppress hyperglycemia-induced proinflammatory genes and alleviate diabetic peripheral neuralgia (Liu et al., 2017). Moreover, miR-590-3p is a novel miRNA involved in multiple diseases. The expression of miR-590-3p is reduced in Alzheimer's disease, suggesting that it may be related to neural degradation (Villa et al., 2011). More importantly, miR-590-3p is down-regulated in DPN model (Gong et al., 2015). However, the specific role and mechanism of miR-590-3p in DPN remain unknown.

RAP1A (Ras-associated protein 1A) is a member of the Ras-like GTPases family and plays a vital role in the cellmatrix and cadherin-mediated cell-cell contacts (Duchniewicz et al., 2006). In addition, RAP1A regulates T cells via augmenting lymphocyte responses and activating integrins (Sebzda et al., 2002). Moreover, inhibition of RAP1A alleviates neuropathic pain ( $\mathrm{Li}$ et al., 2015; Fang et al., 2019). Also, accumulating evidence has proved that pro-inflammatory $\mathrm{T}$ cells migrate into the spinal cord in several pain models, and the immune cell infiltration is involved in diabetic neuropathy (Costigan et al., 2009; Grace et al., 2011; Agarwal et al., 2018). Given all these results, RAP1A might be related to the pathogenesis of DPNP.

The aim of our study was to elucidate the role of $m i \mathrm{R}-590-3 p$ in DPNP. We found that miR-590-3p was downregulated in $\mathrm{db} / \mathrm{db}$ mice (type 2 diabetes model). Overexpression of $m i \mathrm{R}-590-3 p$ alleviated DPNP in $\mathrm{db} /$ $\mathrm{db}$ mice by targeting $\mathrm{R} A P 1 A$, providing strong evidence for the role of altered $m i \mathrm{R}-590-3 p / \mathrm{R} A P 1 A$ axis in the development of DPNP.

\section{MATERIALS AND METHODS}

Animals. All animal experiments were performed in accordance with the Guide for the Care and Use of 
Laboratory Animals and were approved by the Ethics Committee of the First Affiliated Hospital of Soochow University. The 20-week-old male $\mathrm{db} / \mathrm{db}$ mice and $\mathrm{db} / \mathrm{m}$ mice use in this study were purchased from Nanjingjunke (China).

miR-590-3p Agomir and NC agomir (Genepharma, China) were successfully injected into 20-week-old mice according to the manufacturer's protocol. We delivered miR-590-3p agomir or NC agomir to $\mathrm{db} / \mathrm{db}$ mice via tail vein once a week for 4 weeks under anesthesia with isoflurane. Before sacrificing the mice, pain-related behavioral tests were performed. Before the end of the animal experiments, the blood glucose in one drop of tail blood and body weight were determined with a blood glucose monitoring system (Bayer, Germany) and electronic balance (Meitele, Switzerland), respectively. Finally, the dorsal root ganglion (DRG) tissues were extracted, frozen and stored for further experiments.

Isolation of $\mathbf{T}$ cells and transfection. pleens from $\mathrm{db} / \mathrm{m}$ mice were collected and disaggregated, and then the erythrocytes were lysed with Lysing Buffer (BD Biosciences, USA). The $\mathrm{T}$ cells were isolated using $\mathrm{CD}^{+}{ }^{+} \mathrm{T}$ cell Isolation Kit (Miltenyi Biotec, Germany). Next, the $\mathrm{T}$ cells were cultured and stimulated with $0.5 \mu \mathrm{g} / \mathrm{ml}$ anti-CD3e (BD Biosciences, USA) in RPMI-1640 medium (Gibco, USA) containing 10\% fetal bovine serum (FBS; Gibco, USA) at $37^{\circ} \mathrm{C}$.

The $\mathrm{T}$ cells were transfected with pcDNA3 or pcDNA3-RAP1A (GenePharma, China) and miR-590-3p mimic or NC mimic (GenePharma, China) using Lipofectamine 2000 (Invitrogen, USA). The sequences for NC mimic were: 5'-UUCUCCGAACGUGUCACGU-3' and for miR-590-3p mimic: 5'-UAAUUUUAUGUAUAAGCUAGU-3'.

Pain-related behavioral tests. The pain-related behavioral tests were performed as follows: the mice were placed on a hot plate (approx. $55^{\circ} \mathrm{C}$ ), and then the reaction time (latency of the first recoil or struggle of the rear paw) was recorded. To avoid skin damage, we applied a cutoff time of 10 seconds. The shortened withdrawal latency indicated hyperalgesia (Chopra et al., 2010). In addition, each mouse's tail-swinging delay was determined by dipping the distal end of the tail $2-3 \mathrm{~cm}$ into a vessel filled with cold water $\left(10 \pm 1^{\circ} \mathrm{C}\right)$. The data on the duration of the struggle, tail-swinging reaction, or tail-swinging were recorded. The threshold value was set at 15 seconds. A shorter soaking time indicated hyperalgesia which is also attributed to a central mechanism (Kamboj et al., 2010).

qRT-PCR assay. The total RNA from DRG tissues or $\mathrm{T}$ cells was extracted using TRIzol reagent (Takara, Japan), and cDNA was synthesized using PrimeScript ${ }^{\mathrm{TM}}$ RT Master Mix (Takara, Japan). The expression of the target gene was evaluated using SYBR Green PCR Master Mix (Takara, Japan) based on $2^{-\Delta \Delta \mathrm{Ct}}$ method. U6 or $\beta$-actin was used as an endogenous control for the normalization. The primer sequences were as follows: miR-590-3p forward: 5'-AAAGAT'TCCAAGAAGCTAAGGGTG-3' and reverse: 5'-CCTAACTGGT'T'TCCTGTGCCTA-3'; U6 gene forward: 5'-GCT'TCGGCAGCACATATACTAAAAT-3' and U6 gene reverse: 5'-CGCT'TCACGAAT'T'TGCGTGTCAT-3'; RAP1A forward: 5'-TGTCTCACTGCACCTTCA-3' and RAP1A reverse: 5'-GACTTCCCAACGCCTCCT-3'; $\beta$-actin gene forward: 5'-TCACCCACACTGTGCCCATCTACGA-3' and $\beta$-actin gene reverse: 5'-CAGCGGAACCGCTCAT'TGCCAATGG-3'.

Western blot. The cells or DRG tissue were lysed with RIPA lysis buffer (Beyotime, China) to isolate the proteins, and the protein concentration was determined using a BCA kit (Beyotime, China). The equal amount of protein was separated by SDS-PAGE and transferred onto a PVDF membrane. Then the membranes were washed with TBST (Tris Buffered Saline with Tween) buffer three times and incubated with anti-RAP1A antibody (1:800; Abcam, UK) or anti- $\beta$-actin antibody $\left(1: 800 ;\right.$ Abcam, UK) overnight at $4^{\circ} \mathrm{C}$. After washed with TBST buffer for three times, the membranes were then incubated with Goat Anti-Rabbit IgG H\&L (HRP) (1:8000; Abcam, UK) or Goat Anti-Mouse IgG H\&L (HRP) (1:8000; Abcam, UK) for $2 \mathrm{~h}$. Finally, an electrochemiluminescence kit (Beyotime, China) was used to measure the corresponding protein expression levels.

Luciferase reporter assay. The 3'-UTR of RAP1A and the potential target sequences of miR-590-3p were predicted by Targetscan (http://www.targetscan.org) and inserted into pGL3 plasmids (Promega, USA). When isolated $\mathrm{T}$ cells grew to about $80 \%$ confluence, they were co-transfected with pGL3-RAP1A-WT vectors (RAP1A WT) or pGL3-RAP1A-MUT vectors (RAP1A MUT) along with miR-590-3p mimic or NC mimic (GenePharma, China) using Lipofectamine 2000 (Invitrogen, USA). After 24 hours, the luciferase activity was evaluated.

ELISA assay. The levels of IL-1 $\beta$, TNF- $\alpha$ and IL- 6 in DRG tissues were evaluated by ELISA. After the preparation of the samples, the concentration of IL-6, IL-1 $\beta$ and TNF- $\alpha$ in the sample supernatant was determined with IL-6, IL-1 $\beta$ and TNF- $\alpha$ ELISA kit (Elabscience, China), respectively. Each measurement was repeated three times.

Immunohistochemistry (IHC). The collected DRG tissues were subjected to IHC staining. After fixation with $10 \%$ paraformaldehyde, the samples were sliced horizontally, blocked with goat serum (Jackson ImmunoResearch, USA), and probed with anti-CD4 antibody (1:200; Abcam, UK). DAB and hematoxylin were used to stain the tissue slices. RAP1A staining was visualized using a light microscope (Olympus, Japan).

Cell Counting Kit-8 (CCK-8) assay. For CCK-8 assay, after transfection, $\mathrm{T}$ cells were seeded into 96well plates at the density of $3 \times 10^{3}$ cells/well. After cultured for $48 \mathrm{~h}$, the cell proliferation was detected using Cell Counting Kit-8 (CCK-8) assay (Beyotime, China). Finally, the absorbance at the $450 \mathrm{~nm}$ was determined.

Transwell assay. After transfection, $\mathrm{T}$ cells were seeded onto the upper chamber of a Transwell plate (filters diameter: $6.5 \mathrm{~mm}$, pore size: $5 \mu \mathrm{m}$; Corning, USA). The lower chamber was filled with medium containing sphingosine-1-phosphate (S1P; Sigma, USA). After 4 hours of incubation, the cells in the lower chamber were harvested and counted under a light microscope (Olympus, Japan).

Data statistics. The data in this study were analyzed with SPSS 20.0 (SPSS, USA) and presented as mean and Standard Deviation (S.D.). The Student's $t$-test or one-way analysis of variance was performed for comparison between two groups or more than two groups, respectively. $P<0.05$ was considered to indicate a significant difference.

\section{RESULTS}

\section{miR-590-3p was down-regulated in diabetic peripheral neuropathy mice}

Compared to $\mathrm{db} / \mathrm{m}$ mice, the blood glucose in $\mathrm{db} /$ $\mathrm{db}$ mice was markedly elevated, suggesting the suc- 
A

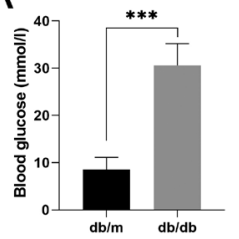

B

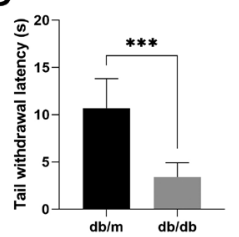

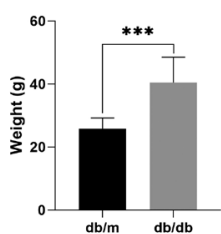
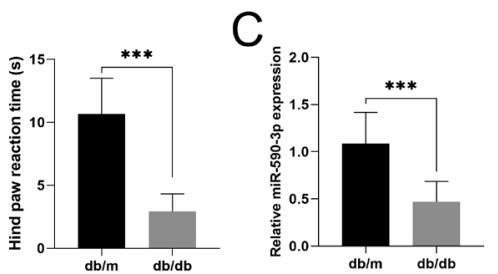

Figure 1. miR-590-3p was down-regulated in diabetic peripheral neuropathy mice

The 20-week-old $\mathrm{db} / \mathrm{db}$ mice (diabetes) and $\mathrm{db} / \mathrm{m}$ mice (control) were studied. (A) Blood glucose and weight were measured. (B) The tail immersion and hot plate tests were performed. (C) The expression of miR-590-3p was determined by qRT-PCR in DRG tissue. Ten mice per group. ${ }^{* * *} p<0.001$.

cessful establishment of diabetes models (Fig. 1A). Besides, compared to $\mathrm{db} / \mathrm{m}$ mice, the body weight was higher in $\mathrm{db} / \mathrm{db}$ mice. For pain-related behavior test, the hind paw reaction time and tail withdrawal latency were lower in $\mathrm{db} / \mathrm{db}$ mice compared to those in $\mathrm{db} / \mathrm{m}$ mice, suggesting that $\mathrm{db} / \mathrm{db}$ mice were in hyperalgesia and suffered from diabetic neuropathy pain (Fig. 1B). Interestingly, compared to $\mathrm{db} / \mathrm{m}$ mice, the $\mathrm{db} / \mathrm{db}$ mice exhibited a reduction of miR-590-3p levels in DRG tissue (Fig. 1C). These results confirmed that diabetic peripheral neuropathy mice models were successfully established and miR-590-3p was down-regulated in diabetic peripheral neuropathy mice.
miR-590-3p alleviated peripheral neuropathic pain in diabetic mice

To further clarify how miR-590-3p alleviates peripheral neuropathic pain in diabetic mice, the 20 -week-old $\mathrm{db} / \mathrm{db}$ mice were injected with miR-590-3p agomir or $\mathrm{NC}$ agomir $(10 \mathrm{mg} / \mathrm{kg})$ weekly for 4 weeks. Compared to $\mathrm{db} / \mathrm{m}$ mice, $m i \mathrm{R}-590-3 p$ level in $\mathrm{db} / \mathrm{db}$ mice was significantly declined, while miR-590-3p agomir markedly promoted miR-590-3p expression compared to NC agomir (Fig. 2A). Moreover, blood glucose and body weight were significantly elevated in $\mathrm{db} / \mathrm{db}$ mice, whereas $m i \mathrm{R}$ 590-3p agomir did not influence blood glucose and body weight (Fig.2B). As for pain-related behavior of mice, the hind paw reaction time and tail withdrawal latency were increased by miR-590-3p agomir, indicating that miR-590-3p agomir alleviated diabetic neuropathy pain (Fig. 2C). Thus, these findings proved that miR-590-3p decreased peripheral neuropathic pain in vivo.

\section{miR-590-3p inhibited the production of pro- inflammatory mediators and neural infiltration by the immune cells}

An excessive amount of blood glucose stimulates the production of pro-inflammatory cytokines (including IL-6, IL-1 $\beta$ and TNF- $\alpha$ ) and directs the cells toward inflammation (Rains \& Jain 2011). When compared to the control, the levels of IL- 6 , IL- $1 \beta$, and TNF- $\alpha$ in $\mathrm{db} / \mathrm{db}$ mice were increased, however, they were significantly reduced by miR-590-3p agomir (Fig. 3A). Accumulating evidence has shown a significant neural infiltration by the immune cells in DPNP patients (Younger et al., 1996; Alexandraki et al., 2006). The infiltration by the immune cells was determined by IHC staining of CD4. The expression of CD4 in DRG tissue of $\mathrm{db} / \mathrm{db}$ mice was elevated (Fig. 3B). After the injection of miR-590-3p agomir into $\mathrm{db} / \mathrm{db}$ mice, the expression of CD4 was reduced (Fig. 3B). These findings proved that exogenous $m i \mathrm{R}-590-3 p$ inhibited the production of pro-inflammatory mediators and neural infiltration by immune cells in $\mathrm{db} / \mathrm{db}$ mice.

\section{A}

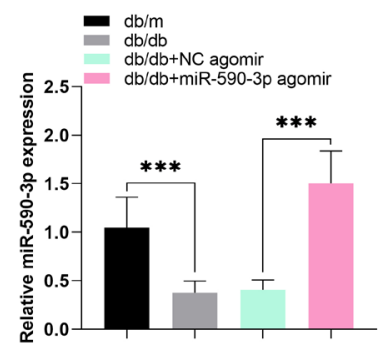

C

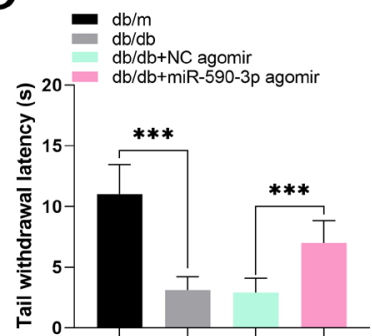

B
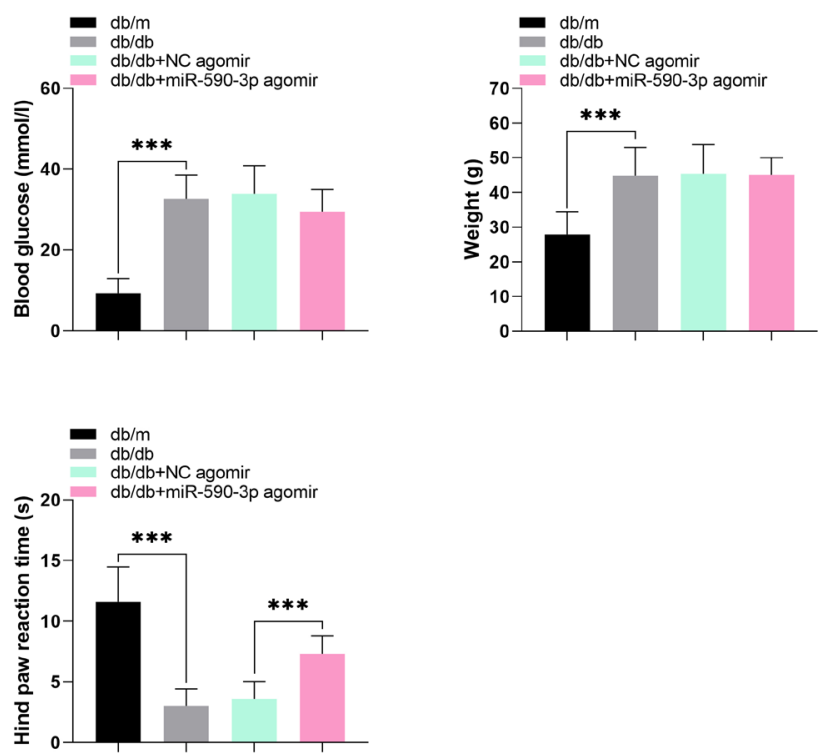

Figure 2. miR-590-3p alleviated peripheral neuropathic pain in diabetic mice

The 20-week-old db/db mice were injected with miR-590-3p agomir or NC agomir (10 mg/kg) weekly for 4 weeks. (A) The expression of miR-590-3p in treated mice was detected by qRT-PCR. (B) Blood glucose and weight were measured. (C) The tail immersion and hot plate tests were performed. Ten mice per group. ${ }^{* *} p<0.01$, ${ }^{* * *} p<0.001$. 
A
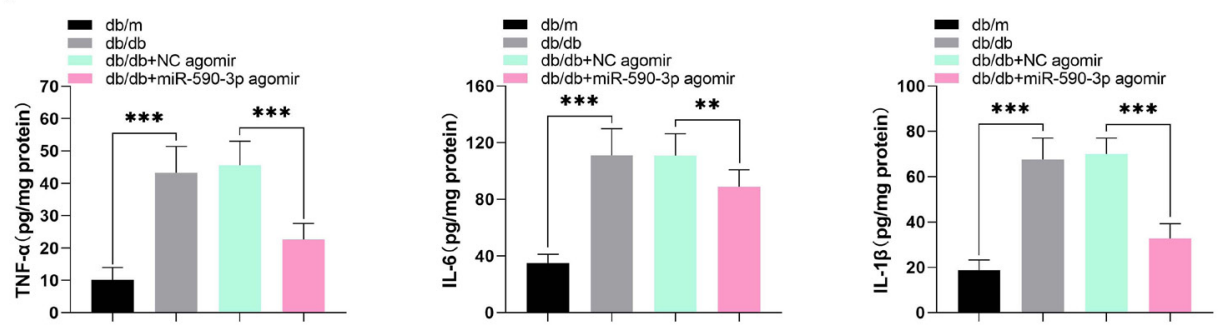

B

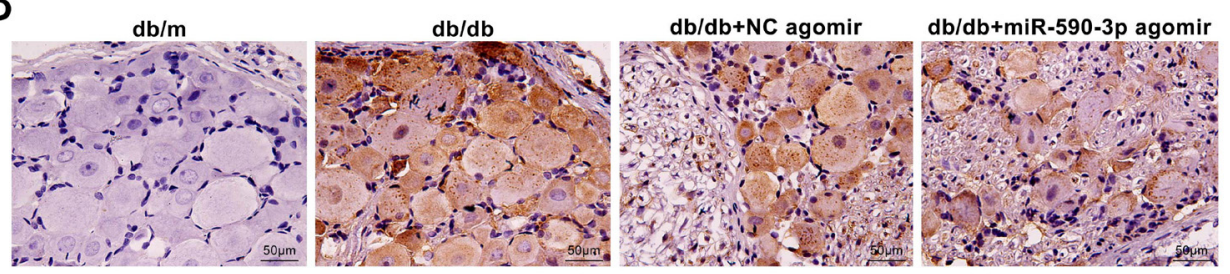

Figure 3. miR-590-3p inhibited the production of pro-inflammatory mediators and neural infiltration by the immune cells

(A) The pro-inflammatory cytokines (such as TNF-a, IL-6, and IL-1 $1 \beta$ ) in differently treated mice were measured by ELISA assay. (B) The expression of CD4 in DRG tissues of differently treated mice was detected by IHC, bar $=100 \mu \mathrm{m} .{ }^{* *} p<0.01,{ }^{* * *} p<0.001$.

A

RAP1A 3. UTR WT

$\operatorname{miR}-590-3 p$

RAP1A 3' UTR MUT

B

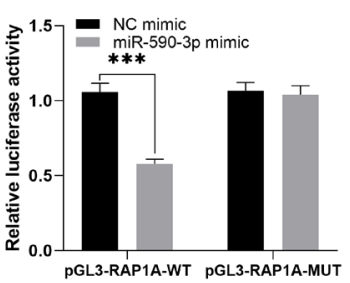

D

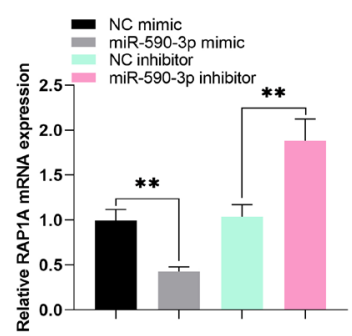

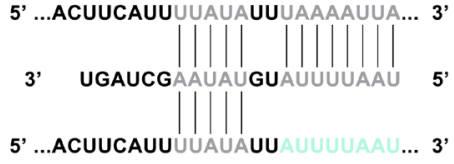

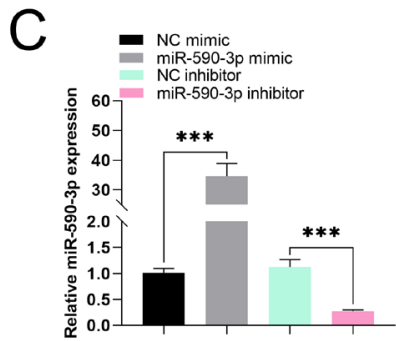

$E$

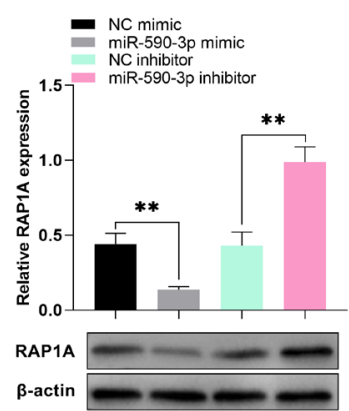

Figure 4. RAP1A was a direct target of $m i R-590-3 p$

(A) The potential miR-590-3p binding sites in the 3'-UTR of RAP1A. (B) Relative luciferase activity of the T cells transfected with miR-590$3 p$ mimic or NC mimic plus the RAP1A-WT or RAP1A-MUT luciferase reporter gene. (C) The efficiency of miR-590-3p mimic or miR-590-3p inhibitor was judged by qRT-PCR assay. (D) The mRNA level of RAP1A was determined by qRT-PCR assay. (E) The protein level of RAP1A was determined by western blot. Every experiment was repeated three times. ${ }^{* *} p<0.01,{ }^{* * *} p<0.001$.

\section{RAP1A was a direct target of miR-590-3p}

Next, the potential target of miR-590-3p was identified. RAP1A, a vital protein, was predicted to be a validated target of miR-590-3p by Targetscan. As illustrated in Fig. 4A, miR-590-3p binds to a conserved site of $\mathrm{RAP1A}$
3'-UTR. The luciferase activity assay confirmed that miR590-3p mimic markedly decreased the luciferase activity of RAP1A 3'-UTR W'T, but not RAP1A 3'-UTR MUT (Fig. 4B). Moreover, miR-590-3p mimic or miR-590-3p inhibitor significantly increased or decreased miR-590-3p expression in T cells (Fig. 4C), respectively. Interestingly, 

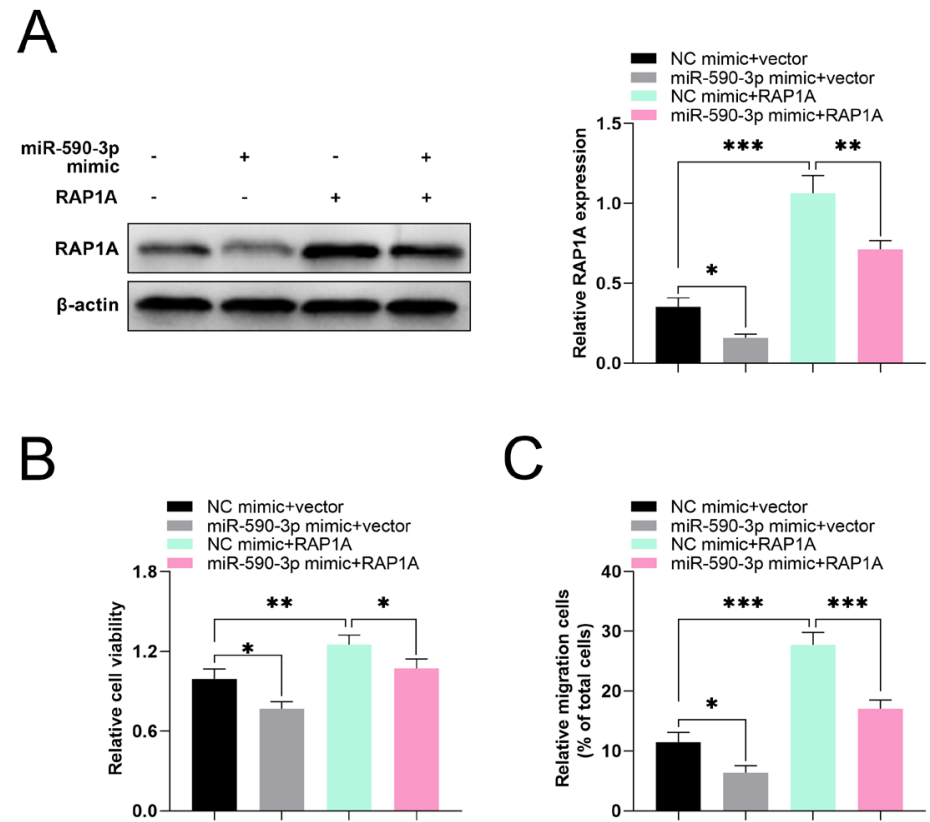

Figure 5. miR-590-3p inhibited T cells proliferation and migration via targeting RAP1A

The RAP1A3-overexpression vector or the corresponding empty vector and NC mimic or miR-590-3p mimic were co-transfected into T cells. (A) The expression of RAP1A was detected by western blot. (B) The cell proliferation was determined using the CCK-8 kit. (C) Four hours after transfection, the cell migration ability was evaluated by Transwell assay. Every experiment was repeated three times. ${ }^{*} p<0.05$, ${ }^{* *} p<0.01,{ }^{* * *} p<0.001$

qRT-PCR and western blot assay confirmed that miR590-3p mimic lowered both mRNA and protein levels of RAP1A in T cells, however, miR-590-3p inhibitor elevated both mRNA and protein levels of RAP1A (Fig. 4D-E). These findings suggested that miR-590-3p regulated the expression of RAP1A via targeting $\mathrm{R} A P 1 A$.

\section{miR-590-3p inhibited proliferation and migration of T cells via targeting RAP1A}

To clarify whether $m i \mathrm{R}-590-3 p$ affected the production of pro-inflammatory mediators and neural immune cells infiltration in a RAP1A-dependent manner, the isolated $\mathrm{T}$ cells were co-transfected with $\mathrm{R} A P 1 A 3$-overexpression vector or the corresponding empty vector and $\mathrm{NC}$ mimic or $m i \mathrm{R}$ 590-3p mimic. Western blot verified that miR-590-3p mimic partially reduced the effect of RAP1A overexpression (Fig. $5 \mathrm{~A})$. More importantly, CCK-8 assay indicated that miR590-3p mimic markedly reduced cell viability, whereas this effect was reversed by overexpression of RAP1A (Fig. 5B). Furthermore, Transwell assay results showed that miR-590$3 p$ mimic reduced cell migration ability, and the effect was reversed by overexpression of RAP1A (Fig. 5C). With these results, we concluded that overexpression of RAP1A partially abrogated the suppressive impact of miR-590-3p on $\mathrm{T}$ cells proliferation and migration.

\section{DISCUSSION}

In the current study, the diabetic peripheral neuropathy mice models were successfully established and we found that miR-590-3p was down-regulated in diabetic peripheral neuropathy mice. Moreover, miR-590-3p agomir reduced the production of pro-inflammatory mediators and neural infiltration by the immune cells in $\mathrm{db} /$ $\mathrm{db}$ mice. Interestingly, $\mathrm{R} A P 1 A$ was predicted to be the direct target of miR-590-3p by Targetscan, and miR-590$3 p$ regulated the expression of $\mathrm{R} A P 1 A$. Finally, the res- cue experiments proved that overexpression of RAP1A partially rescued the suppressive effects of miR-590-3p on $\mathrm{T}$ cells proliferation and migration. Together, these findings suggested that exogenous miR-590-3p may be beneficial to the clinical treatment of DPNP.

The hyperglycemic state may induce the development of DPN, which induces oxidant and inflammatory mediators, thereby leading to deleterious effects in tissues (Anitha et al., 2006; Martyn et al., 2008). Although DPN has been studied, the molecular mechanism underlying inflammatory mediators has not been fully explored (Feldman et al., 2017). Accumulating evidence has confirmed that miRNAs are involved in DPN (Zhu \& Leung 2015). For instance, $m i \mathrm{R}-146 a$ and $m i \mathrm{R}-155$ regulate inflammation in diabetic peripheral neuropathy (Feng et al., 2018; Chen et al., 2019). Meanwhile, $b s a-m i R-590-3 p$ was identified as an LDHAsuppressing microRNA and can significantly improve glucose metabolism in T2D mice model (Chen et al., 2015). Moreover, a recent study demonstrated that miR-590-3p is down-regulated in DR cells (Chen et al., 2018). Similarly, the expression of $m i \mathrm{R}-590-3 p$ was found to be decreased in $\mathrm{db} /$ $\mathrm{db}$ mice, which provided strong evidence for its vital role in diabetes in vivo. Furthermore, miR-590-3p agomir alleviated DPNP in $\mathrm{db} / \mathrm{db}$ mice. Nevertheless, the mechanism of how miR-590-3p alleviates DPNP remains unknown.

Neuropoietic cytokines (such as TNF- $\alpha$, IL-6, and IL-1 $\beta$ ) are imperative to keep homeostasis of peripheral neurons (Skundric \& Lisak 2003). Previous reports demonstrated that IL-1 $\beta, T N F-\alpha$ and IL- 6 levels are elevated in diabetic patients and animals compared to the controls (Abdel Aziz et al., 2001; Lee et al., 2013; Cox et al., 2017). In this study, we demonstrated that miR-590-3p agomir reduced the IL-1 $\beta$, TNF- $\alpha$ and IL- 6 concentrations, and alleviated DPN pain-related behavior in $\mathrm{db} /$ $\mathrm{db}$ mice, indicating that miR-590-3p suppressed DPNP via an anti-inflammatory function. More importantly, diabetes-induced inflammation occurs with inflammatory infiltrations (Thaisetthawatkul et al., 2018). Regarding this, 
we demonstrated that $m i \mathrm{R}-590-3 p$ inhibited neural infiltration by $\mathrm{T}$ cells. Besides, under hyperglycemia, TNF- $\alpha$ production aggravates in neural tissues, thereby inducing nerve damage, and finally resulting in the development of diabetic polyneuropathy (Satoh et al., 2003). Many central nervous system paradigms proved that IL-6 induces axonal regeneration, which may be related to immune cells proliferation and migration (Leibinger et al., 2013; Carmel et al., 2015). Thus, we suspected that miR590-3p affects the $\mathrm{T}$ cells migration and proliferation, which needed more experiment in the next step.

RAP1A is confirmed to be a target of a series of miRNAs and is involved in cell migration and proliferation in non-small cell lung cancer, breast cancer, cervical cancer and prostate cancer (Xiang et al., 2015; Zhang et al., 2018; Cao 2019; Lu et al., 2020). In this study, $R A$ $P 1 A$ was proved to be a direct target of miR-590-3p. As we suspected, miR-590-3p inhibited $\mathrm{T}$ cells proliferation and migration in DPNP, which was reversed by over-expression of RAP1A. These findings suggested that miR590-3p inhibited $\mathrm{T}$ cells proliferation and migration via targeting $\mathrm{R} A P 1 A$ in DPNP, which was consistent with the previous research. A recent study pointed out that IncMIR205HG acts as a natural decoy for miR-590-3p and leads to the process of head and neck squamous cell carcinoma (Di Agostino et al., 2018). Besides, RAP1A contributes to cell migration via regulating the MAPK/ERK pathway (Fujita et al., 2005; Zhang et al., 2018). Given all these reports, we hypothesized that IncMIR205HG may target $m i R-590-3 p$ and activate the MAPK/ERK pathway in DPNP. However, this hypothesis needs more experiments to confirm it in the future.

In summary, our data demonstrated for the first time that miR-590-3p inhibited the production of proinflammatory mediators and neural infiltration by $\mathrm{T}$ cells in DPNP. Moreover, additional in vitro studies indicated that miR-590-3p inhibited $\mathrm{T}$ cells proliferation and migration by targeting $\mathrm{RAP} 1 A$. Our findings provide a novel insight into the molecular mechanism and molecular basis of treating DPN with exogenous miR-590-3p. Although the treatment strategy of exogenous miR-590-3p can be implemented, the interaction network of the miR590-3p in diabetes remains to be elucidated.

\section{Acknowledgements}

Not applicable.

\section{Funding}

Not applicable.

\section{Competing interests}

The authors state that there are no conflicts of interest to disclose.

\section{Ethics approval}

All animal experiments were in accordance with the Guide for the Care and Use of Laboratory Animals and were approved by the Ethics Committee of the First Affiliated Hospital of Soochow University.

\section{Statement of Informed Consent}

Not applicable.

\section{Availability of data and materials}

All data generated or analyzed during this study are included in this published article.

\section{Authors' contributions}

Yihua $\mathrm{Wu}$ and Ye Gu designed the study, supervised the data collection, analyzed the data, Bimin Shi interpreted the data and prepared the manuscript for publication, supervised the data collection, analyzed the data and reviewed the draft of the manuscript. All authors have read and approved the manuscript.

\section{REFERENCE}

Abdel Aziz MT, Fouad HH, Mohsen GA, Mansour M, Abdel Ghaffar S (2001) TNF-alpha and homocysteine levels in type 1 diabetes mellitus. East Mediterr Health J 7: 679-688

Agarwal N, Helmstadter J, Rojas DR, Bali KK, Gangadharan V, Kuner $\mathrm{R}$ (2018) Evoked hypoalgesia is accompanied by tonic pain and immune cell infiltration in the dorsal root ganglia at late stages of diabetic neuropathy in mice. Mol Pain 14: 1744806918817975 . https:// doi.org/10.1177/1744806918817975

Alexandraki K, Piperi C, Kalofoutis C, Singh J, Alaveras A, Kalofoutis A (2006) Inflammatory process in type 2 diabetes: The role of cytokines. Ann N Y Acad Sci 1084: 89-117. https://doi.org/10.1196/ annals.1372.039

Anitha M, Gondha C, Sutliff R, Parsadanian A, Mwangi S, Sitaraman SV, Srinivasan S (2006) GDNF rescues hyperglycemia-induced diabetic enteric neuropathy through activation of the PI3K/Akt pathway. I Clin Invest 116: 344-356. https://doi.org/10.1172/JCI26295

Boulton AJ, Malik RA, Arezzo JC, Sosenko JM (2004) Diabetic somatic neuropathies. Diabetes Care 27: 1458-1486. https://doi.org/10.2337/ diacare.27.6.1458

Calcutt NA (2004) Experimental models of painful diabetic neuropathy. J Neurol Sci 220: 137-139. https://doi.org/10.1016/j.jns.2004.03.015

Cao XM (2019) Role of miR-337-3p and its target RAP1A in modulating proliferation, invasion, migration and apoptosis of cervical cancer cells. Cancer Biomark 24: 257-267. https://doi.org/10.3233/ CBM-181225

Carmel JB, Young W, Hart RP (2015) Flipping the transcriptional switch from myelin inhibition to axon growth in the CNS. Front Mol Neurosci 8: 34. https://doi.org/10.3389/fnmol.2015.00034

Chen J, Liu W, Yi H, Hu X, Peng L, Yang F (2019) MicroRNA-155 mimics ameliorates nerve conduction velocities and suppresses hyperglycemia-induced pro-inflammatory genes in diabetic peripheral neuropathic mice. Am J Transl Res 11: 3905-3918

Chen R, M Chen, Xiao Y, Liang Q, Cai Y, Chen L, Fang M (2018) Bioinformatics analysis of microRNAs related to blood stasis syndrome in diabetes mellitus patients. Biosci Rep 38: https://doi. org/10.1042/BSR20171208

Chen Y, Wang X, Shao X (2015) A combination of human embryonic stem cell-derived pancreatic endoderm transplant with LDHA-repressing miRNA can attenuate high-fat diet induced type II diabetes in mice. J Diabetes Res 2015: 796912. https://doi. org/10.1155/2015/796912

Chopra K, Tiwari V, Arora V, Kuhad A (2010) Sesamol suppresses neuro-inflammatory cascade in experimental model of diabetic neuropathy. J Pain 11: 950-957. https://doi.org/10.1016/j. jpain.2010.01.006

Costigan M, Moss A, Latremoliere A, Johnston C, Verma-Gandhu M, Herbert TA, Barrett L, Brenner GJ, Vardeh D, Woolf CJ, Fitzgerald M (2009) T-cell infiltration and signaling in the adult dorsal spinal cord is a major contributor to neuropathic pain-like hypersensitivity. J Neurosci 29: 14415-14422. https://doi.org/10.1523/JNEUROSCI.4569-09.2009

Cox AA, Sagot Y, Hedou G, Grek C, Wilkes T, Vinik AI, Ghatnekar G (2017) Low-dose pulsatile interleukin-6 as a treatment option for diabetic peripheral neuropathy. Front Endocrinol (Lausanne) 8: 89. https://doi.org/10.3389/fendo.2017.00089

Davies M, Brophy S, Williams R, Taylor A (2006) The prevalence, severity, and impact of painful diabetic peripheral neuropathy in type 2 diabetes. Diabetes Care 29: 1518-1522. https://doi.org/10.2337/ dc05-2228

Di Agostino S, Valenti F, Sacconi A, Fontemaggi G, Pallocca M, Pulito C, Ganci F, Muti P, Strano S, Blandino G (2018) Long non-coding MIR205HG depletes Hsa-miR-590-3p leading to unrestrained proliferation in head and neck squamous cell carcinoma. Theranostics 8 : 1850-1868. https://doi.org/10.7150/thno.22167

Duchniewicz M, Zemojtel T, Kolanczyk M, Grossmann S, Scheele JS, Zwartkruis FJ (2006) RAP1A-deficient T and B cells show impaired integrin-mediated cell adhesion. Mol Cell Biol 26: 643-653. https:// doi.org/10.1128/MCB.26.2.643-653.2006

Fang B, Wei L, Dong K, Niu X, Sui X, Zhang H (2019) miR-202 modulates the progression of neuropathic pain through targeting RAP1A. J Cell Biochem 120: 2973-2982. https://doi.org/10.1002/ jcb. 27025 
Feldman EL, KA Nave, TS Jensen,DLH Bennett (2017) New Horizons in Diabetic Neuropathy: Mechanisms, Bioenergetics, and Pain. Neuron. 93: 1296-1313. https://doi.org/10.1016/j.neuron.2017.02.005

Feng Y, Chen L, Luo Q, Wu M, Chen Y, Shi X (2018) Involvement of microRNA-146a in diabetic peripheral neuropathy through the regulation of inflammation. Drug Des Devel Ther 12: 171-177. https:// doi.org/10.2147/DDDT.S157109

Fujita H, Fukuhara S, Sakurai A, Yamagishi A, Kamioka Y, Nakaoka Y, Masuda M, Mochizuki N (2005) Local activation of Rap1 contributes to directional vascular endothelial cell migration accompanied by extension of microtubules on which RAPL, a Rap1-associating molecule, localizes. J Biol Chem 280: 5022-5031. https://doi. org/10.1074/jbc.M409701200

Gong Q, Lu Z, Huang Q, Ruan L, Chen J, Liang Y, Wang H, Yue Y, Feng S (2015) Altered microRNAs expression profiling in mice with diabetic neuropathic pain. Biochem Biophys Res Commun 456: 615-620. https://doi.org/10.1016/j.bbrc.2014.12.004

Grace PM, Rolan PE, Hutchinson MR (2011) Peripheral immune contributions to the maintenance of central glial activation underlying neuropathic pain. Brain Behav Immun 25: 1322-1332. https://doi. org/10.1016/j.bbi.2011.04.003

Kamboj SS, Vasishta RK, Sandhir R (2010) N-acetylcysteine inhibits hyperglycemia-induced oxidative stress and apoptosis markers in diabetic neuropathy. I Neurochem 112: 77-91. https://doi.org/10.1111/ j.1471-4159.2009.06435.x

Lee KA, Jin HY, Baek HS, Park TS (2013) The protective effects of DA-9801 (Dioscorea extract) on the peripheral nerves in streptozotocin-induced diabetic rats. J Nutr Sci Vitaminol (Tokyo) 59: 437-446. https://doi.org/10.3177/jnsv.59.437

Leibinger M, Muller A, Gobrecht P, Diekmann H, Andreadaki A, Fischer D (2013) Interleukin-6 contributes to CNS axon regeneration upon inflammatory stimulation. Cell Death Dis 4: e609. https://doi. org/10.1038/cddis.2013.126

Li H, Huang Y, Ma C, Yu X, Zhang Z, Shen L (2015) MiR-203 involves in neuropathic pain development and represses Rap1a expression in nerve growth factor differentiated neuronal PC12 cells. Clin J Pain 31: 36-43. https://doi.org/10.1097/AJP.0000000000000070

Liu XS, Fan B, Szalad A, Jia L, Wang L, Wang X, Pan W, Zhang L, Zhang R, Hu J, M Zhang X, Chopp M, Zhang ZG (2017) MicroRNA-146a Mimics reduce the peripheral neuropathy in type 2 diabetic mice. Diabetes 66: 3111-3121. https://doi.org/10.2337/db16-1182

Lu J, Zhou L, Wu B, Duan Y, Sun Y, Gu L, Xu D, Du C (2020) MiR501-3p functions as a tumor suppressor in non-small cell lung cancer by downregulating RAP1A. Exp Cell Res 387: 111752. https:// doi.org/10.1016/j.yexcr.2019.111752

Martyn JA, Kaneki M, Yasuhara S (2008) Obesity-induced insulin resistance and hyperglycemia: etiologic factors and molecular mechanisms. Anesthesiology 109: 137-148. https://doi.org/10.1097/ ALN.0b013e3181799d45

Rains JL, Jain SK (2011) Oxidative stress, insulin signaling, and diabetes. Free Radic Biol Med 50: 567-575. https://doi.org/10.1016/j. freeradbiomed.2010.12.006
Satoh J, Yagihashi S, Toyota T (2003) The possible role of tumor necrosis factor-alpha in diabetic polyneuropathy. Exp Diabesity Res 4: 65-71. https://doi.org/10.1155/EDR.2003.65

Sebzda E, Bracke M, Tugal T, Hogg N, Cantrell DA (2002) RA RAP1A positively regulates $T$ cells via integrin activation rather than inhibiting lymphocyte signaling. Nat Immunol 3: 251-258. https:// doi.org/10.1038/ni765

Skundric DS, Lisak RP (2003) Role of neuropoietic cytokines in development and progression of diabetic polyneuropathy: from glucose metabolism to neurodegeneration. Exp Diabesity Res 4: 303-312. https://doi.org/10.1155/EDR.2003.303

Thaisetthawatkul P, Fernandes JA Jr., Piccione E, Truong L, Dyck PJB (2018) Inflammatory Diabetic Neuropathy: Helpful Diagnostic Parameters. J Clin Neuromuscul Dis 20: 7-13. https://doi.org/10.1097/ CND.0000000000000207

Vanotti A, Osio M, Mailland E, Nascimbene C, Capiluppi E, Mariani C (2007) Overview on pathophysiology and newer approaches to treatment of peripheral neuropathies. CNS Drugs 21 (Suppl 1): 3-12; discussion 45-16. https://doi.org/10.2165/00023210-20072100100002

Villa C, Fenoglio C, De Riz M, Clerici F, Marcone A, Benussi L, Ghidoni R, Gallone S, Cortini F, Serpente M, Cantoni C, Fumagalli G, Martinelli Boneschi F, Cappa S, Binetti G, Franceschi M, Rainero I, Giordana MT, Mariani C, Bresolin N, Scarpini E, Galimberti D (2011) Role of hnRNP-A1 and miR-590-3p in neuronal death: genetics and expression analysis in patients with Alzheimer disease and frontotemporal lobar degeneration. Rejuvenation Res 14: 275-281. https://doi.org/10.1089/rej.2010.1123

Wu W, Yang J, Feng X, Wang H, Ye S, Yang P, Tan W, Wei, Zhou Y (2013) MicroRNA-32 (miR-32) regulates phosphatase and tensin homologue (PTEN) expression and promotes growth, migration, and invasion in colorectal carcinoma cells. Mol Cancer 12: 30. https://doi.org/10.1186/1476-4598-12-30

Xiang J, Bian C, Wang H, Huang S, Wu D (2015) MiR-203 downregulates RAP1A and suppresses cell proliferation, adhesion and invasion in prostate cancer. J Exp Clin Cancer Res 34: 8. https://doi. org/10.1186/s13046-015-0125-x

Younger DS, Rosoklija G, Hays AP, Trojaborg W, Latov N (1996) Diabetic peripheral neuropathy: a clinicopathologic and immunohistochemical analysis of sural nerve biopsies. Muscle Nerve 19: 722-727. https://doi.org/10.1002/(SICI)1097-4598(199606)19:6<722::AIDMUS6>3.0.CO;2-C

Zhang T, Jiang K, Zhu X, Zhao G, Wu H, Deng G, Qiu C (2018) miR-433 inhibits breast cancer cell growth via the MAPK signaling pathway by targeting Rap1a. Int J Biol Sci 14: 622-632. https://doi. org $/ 10.7150 /$ iibs. 24223

Zhang Y, Song C, Liu J, Bi Y, Li H (2018) Inhibition of miR-25 aggravates diabetic peripheral neuropathy. Neuroreport 29: 945-953. https://doi.org/10.1097/WNR.0000000000001058

Zhu H, Leung SW (2015) Identification of microRNA biomarkers in type 2 diabetes: a meta-analysis of controlled profiling studies. Diabetologia 58: 900-911. https://doi.org/10.1007/s00125-015-3510-2 